\title{
Propuesta de intervención en el CEIP Meseta de Orcasitas de Madrid para el fomento y el desarrollo de los derechos humanos en sexto curso de Educación Primaria
}

\author{
Neubauer Esteban, Adrián \\ Grupo de Investigación sobre Políticas Educativas Supranacionales, Madrid, España \\ Adrian.neubauer@outlook.com
}

\section{Resumen}

En el CEIP Meseta de Orcasitas, situado en el barrio de Orcasitas, Madrid, se ha iniciado un cambio metodológico en sexto curso de Educación Primaria, concretamente, en la asignatura de «Valores». El objetivo de esta nueva metodología es fomentar y desarrollar los derechos humanos, siguiendo una metodología reflexiva, dialógica y lúdica.

La estructura que ha seguido el proceso ha totalmente democrática con el alumnado, desde la elección de la temática y los contenidos, hasta los objetivos, metodología y evaluación. Los objetivos que concretó el alumnado fueron: «1) transmitir nuestro aprendizaje a todo el colegio; 2) respetar a todo el mundo; 3 ) trabajar especialmente la igualdad de género; y por último, 4) conocer y combatir los prejuicios en sexto de Primaria». Por otro lado, la metodología elegida fueron actividades como: vídeos, juegos, trabajos individuales y grupales, charlas de expertos externos, hacer una obra de teatro y el uso de la red social Instagram como medio de difusión de sus actividades y aprendizajes. Además, la evaluación se llevará a cabo a través de una autoevaluación y coevaluación con los compañeros y compañeras de clase.

En conclusión, este proyecto persigue favorecer una mayor conciencia crítica, autonomía y la capacidad de trabajar en equipo, de modo que puedan transferir esas competencias al resto de asignaturas y a su día a día, con el fin de generar un impacto en su ciudadanía y derechos adheridos a esta condición, tales como la libertad de expresión y la participación.

\section{Abstract}

In the neighbourhood of Orcasitas, Madrid, it is been a methodological change in the CEIP Meseta de Orcasitas, concretely, in sixth grade of Primary Education in "Values» subject. The objective of this new methodology is to foment and develop human rights, following a reflexive, dialogical and playful methodology. The project has followed a democratic process with pupils, since thematic election and contents, to objective, methodology and evaluation. The objectives that concrete pupils were: «1) To transmit our learning to all the school; 2) To respect everyone; 3) To work specially gender equality; 4) To know and fight against prejudices in sixth grade». On the other hand, methodology includes activities as: videos, games, individual and work jobs, speeches of experts, making a play theatre and the use of Instagram to transmit their activities and learning. Further, evaluation will be a done by a coevaluation and autoevaluation with classmates. In conclusion, this project wants to favour more critical awareness, autonomy and capacity of work in team, so they could transfer their competences to the rest of subjects and in their day to day, with the objective to generate an impact in their citizenship and rights, as expression liberty and participation.

Palabras clave: Derechos Humanos, Educación Primaria, Intervención educativa, Metodología.

Keywords: Human rights, Primary education, Educative intervention, Methodology.

\section{INTRODUCCIÓN}

En el madrileño barrio de Usera se encuentra el CEIP Meseta de Orcasitas, un colegio público de línea 3, a excepción del curso de 3 años de Educación Infantil, que tiene tan sólo 2 líneas. En este centro se inició en el mes de Diciembre un proyecto sobre los derechos humanos en el grupo de $6^{\circ} \mathrm{A}$ de Educación Primaria.

Este proyecto tiene como objetivo principal que el alumnado conozca y promueva los derechos humanos en el centro educativo. Para ello, el docente creará y favorecerá espacios y actividades que inciten a la reflexión y al debate entre los discentes.

http://doi.org/10.25145/c.educomp.2018.16.089 
En este trabajo, se presentarán las actividades realizadas por este grupo de alumnos y alumnas, así como su opinión y sentimientos respecto al proyecto.

\section{OBJETIVOS DEL PROYECTO}

A la hora de dar comienzo a este proyecto, el alumnado se reunió en una asamblea donde todos participaron y pudieron dar su opinión para concretar los objetivos del proyecto. Previamente, el docente les explicó en qué consistía un objetivo, catalogándolo como «algo que quieres conseguir». Una vez tomada conciencia del término, mediante un intercambio de ideas y la posterior votación democrática sobre las mismas, se acordaron cinco objetivos:

1. Aprender los derechos humanos.

2. Transmitir nuestro aprendizaje a todo el colegio.

3. Respetar a todos los compañeros y compañeras de clase.

4. Trabajar y conocer especialmente la igualdad de género.

5. Conocer y combatir los prejuicios en sexto de primaria.

Los objetivos citados anteriormente son las propias del alumnado, sin embargo, el docente se propuso que el alumnado «adquiriera una mayor conciencia sobre las desigualdades existentes en el mundo».

\section{METODOLOGÍA}

Para recopilar la opinión del alumnado sobre el proyecto se elaboró un cuestionario a través de la plataforma de Google, con el fin de sistematizar las respuestas del alumnado con la mayor objetividad posible.

El alumnado, compuesto por quince alumnos (6 chicas y 9 chicos) respondió al cuestionario de forma simultánea, mientras el docente explicaba a una a una las preguntas del formulario, facilitando así su comprensión y su respuesta. Dispusieron de un tiempo total de treinta minutos para cumplimentar las 19 preguntas del mismo.

Las cinco primeras estaban destinadas a saber en qué grado se habían alcanzado los cinco objetivos propuestos para este proyecto. Por otro lado, las siguientes once preguntas tenían como objetivo que el alumnado valorase y reflexionase sobre la utilidad de este proyecto respecto a sus competencia lingüística, su capacidad crítica, su trabajo en equipo y sus sentimientos en el aula. Para terminar, en las dos últimas preguntas el alumnado pudo expresar los posibles aspectos de mejora y puntos fuertes del proyecto.

\section{PROPUESTA DE INTERVENCIÓN}

En este proyecto educativo se llevaron a cabo una serie de actividades con el fin de alcanzar los objetivos del alumnado y del maestro. En la misma asamblea que se establecieron los objetivos, se les preguntó a los discentes, «de qué modo podrían conseguir los objetivos, es decir, qué harían para cumplir lo que quieren». De esas opiniones, concluyeron que querían recibir clases magistrales por parte del docente, aunque no sería el único recurso pedagógico. Además, apostaron por el visionado de vídeos y realizar juegos relacionados con la materia. También, quisieron diseñar y elaborar carteles y fotografías para mostrar al centro todo su aprendizaje.

En esta línea, también mencionaron la posibilidad de que algún miembro familiar o experto sobre el tema, pudiese venir a explicarles con mayor profundidad las cuestiones trabajadas. Sin embargo, la gran propuesta que realizaron, fue la de preparar y llevar a cabo exposiciones y presentaciones por todo el centro, especialmente para el alumnado más pequeño, con el fin de acercar y promover el conocimiento de los derechos humanos de un modo lúdico y sencillo. 
Siguiendo esta idea, también hablaron de la posibilidad de realizar una obra de teatro en lugar de exposiciones.

Para terminar, cabe destacar la importancia y el deseo que le otorgó el alumnado a la elaboración de resúmenes, trabajados individuales y grupales.

A raíz de estas propuestas, el docente planificó una serie de actividades que vamos a detallar en mayor profundidad y detalle en las siguientes líneas:

\subsection{Taller sobre igualdad de género}

Tras una breve introducción por parte del maestro sobre los derechos humanos y su origen, acudió al centro educativo Rafael Mesto, abogado que actualmente está cursando el máster en Derechos Humanos en la Universidad Carlos III de Madrid. Durante esta sesión, les entregó a los alumnos diferentes profesiones (médico, maestra, piloto, azafata...) que debían categorizar como «masculinas» o «femeninas». Una vez realizada la primera parte del taller, tenían que poner al lado de la profesión si creían que era un empleo bien remunerado, medio o mal remunerado.

Las conclusiones a las que llegó el alumnado en este taller fue que los hombres tienen un mayor abanico de empleos y que además, están mejo remunerados que el de las mujeres, siendo evidente la desigualdad de género en el mundo laboral.

\subsection{Los derechos humanos en emoticonos}

El docente les hace entrega a cada alumno de una Declaración Universal de los Derechos Humanos (1948) adaptada y simplificada para el lenguaje de un niño de Primaria. Tras leer y explicar dicha Declaración, el docente les presenta un ejemplo de cómo podemos representar un derecho humano mediante simples emoticonos de Whatsapp. Una vez comprendida la actividad pro el alumnado, toda la clase, trabajando conjuntamente, han de elaborar una Declaración con tan sólo emoticonos de Whatsapp. Para ello, el docente les facilita varios folios tamaño DIN-3 con un gran número de emoticonos, todos ellos repetidos. El tiempo establecido para la entrega de la actividad es de cuatro sesiones de 45 minutos.

\subsection{Análisis crítico de los titulares de prensa}

Siguiendo la idea de que todo discurso trae consigo una carga ideológica, negando de tal modo un discurso neutro (González, N. F., 2015) y acercando al alumnado al análisis crítico del discurso (Martín Rojo, L., 2006), el maestro les hace entrega de pequeños titulares de noticias sacados de la página web de ACNUR. En estas pequeñas noticias plastificadas del tamaño de una carta de naipes, se vislumbra claramente un titular, una imagen de la noticia y la publicación. De forma individual han de analizar qué derechos humanos han podido ser violados potencialmente tras leer solamente el titular. El tiempo para esta actividad fue de tan sólo una sesión de 45 minutos.

\subsection{Búsqueda de violaciones de los derechos humanos en el mundo y propuesta de solución}

Para esta actividad se decidió juntar a las clases de $6^{\circ} \mathrm{A}$ y $6^{\circ} \mathrm{C}$, pues era deseo expreso del alumnado el trabajar conjuntamente. En grupos compuestos por discentes de ambas clases, debían buscar una noticia en la que se violasen los derechos humanos a través del ordenador de las aulas. Una vez encontrada, debían elaborar una cartulina en la que resumieran la noticia, señalaran qué derechos humanos se estaban violando y cómo podrían solucionarlo, ofreciendo soluciones reales y factibles. El tiempo total para esta actividad fue de dos sesiones. 


\title{
3.5. Salvar al huevo Ryan
}

En grupos de cuatro personas debían elaborar una estructura que les permitiera tirar un huevo desde un segundo piso sin que éste se rompiera. La construcción de la estructura se realizó con materiales reciclados y en tres sesiones. Esta actividad pese a no ser específica a priori para trabajar los derechos humanos, sí que perseguía desarrollar la capacidad de mantener un diálogo y resolver los problemas de manera pacífica. Además, esta tarea favoreció la cohesión grupal gracias a su carácter lúdico.

\subsection{Taller de fotografía a favor de la igualdad de género y los derechos humanos}

A la luz del trabajo de Lucía Godoy (2017), se decidió llevar a cabo un taller de fotografía en el aula, dado su potencial artístico y su contribución a las competencias digitales. En una primera sesión el alumnado decidió mediante una asamblea qué fotografías realizarían (lugar, modelos, cómo posarían...). Más tarde, el docente les proporcionó una cámara para poder tomar las fotografías pertinentes. Contaron con un total de tres sesiones para tomar las fotografías por todo el recinto escolar (cocina, gimnasio, sala de fotocopias...).

Una vez realizadas, el alumnado decidió qué doce fotografías eran las que más les gustaba. Posteriormente, fueron impresas y expuestas en el hall del centro educativo para mostrar al resto del colegio su aprendizaje, y con ello, concienciar sobre la importancia de respetar los derechos humanos y defender la igualdad de género.

\subsection{Elaboración de una entrevista para conocer los prejuicios de sus compañeros}

\begin{abstract}
Esta actividad, a la igual que la posterior, no ha sido llevada a cabo hasta la fecha, aunque está programada para ser realizada en este curso académico (2017-2018). Sin embargo, podemos explicar brevemente cuáles son las ideas que tiene el alumnado para su puesta en marcha. Mediante la herramienta de "Google encuestas», elaborarán una encuesta para conocer los prejuicios de sus compañeros de todo sexto. Entregarán la dirección (URL) para acceder a la encuesta, y de forma anónima responderán en sus casas. Una vez recogidos los datos, pasarán a analizar los datos obtenidos.
\end{abstract}

\subsection{Presentación a favor de los derechos humanos en la escuela}

A priori, el alumnado realizará una presentación de Power Point en el que de forma sencilla contarán en qué consisten los derechos humanos y cuáles son. El trabajo será dividido por los propios alumnos, de modo que unos se encarguen de la elaboración de la presentación oral, otros de las diapositivas y otros del contenido de las mismas. Estas presentaciones se realizarán en tantas clases y cursos como sea posible.

\section{RESULTADOS Y OPINIÓN DEL ALUMNADO}

Una vez analizados los cuestionarios, en los cinco primeros ítems se pudo discernir un gran consenso respecto al grado de cumplimiento de los cuatro primeros, mientras que en el quinto por el contrario, existe una gran disparidad de respuestas y opiniones.

De forma más específica, el alumnado concluyó mayoritariamente, que se había cumplido dicho objetivo, pues el 50 \% marcó la casilla de "totalmente de acuerdo», seguido de un 43,8 \% que decía estar «de acuerdo». Sin embargo, el 6,2\% de las respuestas se mostraba en desacuerdo respecto a esta afirmación. 
El segundo ítem tiene una rotunda respuesta negativa ante el escaso cumplimiento de este objetivo, con un claro $93,8 \%$ de votos en esta línea.

En cuanto al objetivo de «respetar a todo el mundo», es posible vislumbrar cómo la amplia mayoría coincide en que éste se ha cumplido, con un apoyo total del $37,5 \%$ y del $56,5 \%$ de acuerdo. Esta tendencia se mantiene en cuanto al cuarto objetivo, pues el $93,8 \%$ afirma que se ha trabajado especialmente la igualdad de género.

El quinto objetivo sin embargo, muestra una clara divergencia en los resultados, pues el $50 \%$ de la clase opina que sí se han conocido y combatido los prejuicios en sexto de Primaria, y el otro $50 \%$ opina de forma contraria.

Por otro lado, en cuanto a las competencias adquiridas durante este proyecto cabe destacar que el $80,3 \%$ considera que ahora es más capaz de mantener un debate que al principio del proyecto, además de ser capaz de presentar y exponer sus ideas y opiniones de forma ordenada. Esto que se relaciona directamente con que todos ellos y ellas han aprendido a resolver los problemas de manera pacífica a través del diálogo. Este hecho ha sido favorecido también por la mejora en su capacidad para trabajar en equipo $(80,3 \%)$ y la mejora en la confianza y seguridad a la hora de presentar sus ideas ante sus compañeros y compañeras (75\%). En esta línea, el $80,3 \%$ afirma que este proyecto ha servido para mejorar la relación con sus compañeros.

Respecto a las actividades, el $93,8 \%$ del alumnado considera que son divertidas y motivantes, por lo que todos ellos se encuentran más motivados con la asignatura respecto al inicio del proyecto.

Otro aspecto positivo de este proyecto es que el 93,8\% tiene mayor conciencia y compromiso con los derechos humanos y que además son capaces de identificar conductas machistas o que promueven la desigualdad de género $(87,5 \%)$. Además, la gran mayoría siente que puede expresar su opinión libremente $(93,8 \%)$.

En cuanto a los aspectos de mejora, la mayoría reclama una mayor utilización de vídeos y de las redes sociales (Instagram). Además, un pequeño grupo reclama mayor compromiso y participación por parte de sus compañeros y compañeras.

Para terminar, el alumnado señala que los aspectos más positivos de dicho proyecto son su componente lúdico y motivante, gracias a la metodología y actividades propuestas, destacando el taller de fotografía y los «emojis», tal y como afirma un alumno.

El alumnado valora muy positivamente la libertad de expresión y organización que les brinda el docente a la hora de desarrollar las actividades. Cabe destacar la importancia y el valor que le dan al trabajo en grupo y a la posibilidad de evaluar al docente al final de cada evaluación. En último lugar, la gran mayoría coinciden en que el tema del proyecto es «muy interesante».

\section{DISCUSIÓN Y CONCLUSIONES}

Este proyecto ha tenido un impacto muy positivo para el alumnado del CEIP Meseta de Orcasitas, pues la gran mayoría de ellos se sienten más confiados, más motivados, tienen más herramientas para resolver problemas y además, conocen mejor y están más comprometidos con los derechos humanos.

La metodología aquí propuesta puede suponer un reto para el alumnado al comienzo del proyecto, pues la gran libertad y autonomía que se les otorga puede generar momentos de dudas y desconcierto por parte del alumnado respecto a la tarea requerida. Sin embargo, este aprendizaje es de mayor calidad y significatividad, pues la implicación del alumnado y el grupo es total a nivel emocional e intelectual.

En definitiva, es preciso reivindicar y fortalecer los derechos humanos desde la escuela pública, acorde a la legislación educativa vigente, y debe hacerse acorde a éstos mismos, pues una educación que no esté fundamentada sobre estos derechos, no es educación (Tomasevski, K., 2004). 


\section{REFERENCIAS BIBLIOGRÁFICAS}

Godoy, L. (2017). Fotografías en el aula: ¿nuevas prácticas de lectura y escritura mediadas por TIC?. Quaderns digitals: Revista de Nuevas Tecnologías y Sociedad, (85), 9.

Gonzalez, N. F. (2015). PISA como instrumento de legitimación de la reforma de la LOMCE. Bordón. Revista de pedagogía, 67(1), 165-178.

Martín Rojo, L. (2006). El análisis crítico del discurso. Fronteras y exclusión social en los discursos racistas. En L. Íñigue (ed.), Análisis del discurso. Manual para las ciencias sociales (pp. 161-196). Barcelona: UOC.

Tomasevski, K. (2004). Indicadores del derecho a la educación. Revista lidh, 40, 341-388. 